\title{
Satisfação de negros e não negros assistidos por Centros de Atenção Psicossocial em Álcool e Outras Drogas
}

\author{
Satisfaction of black and non-black individuals assisted at the Alcohol \\ and Drugs Psychosocial Care Center
}

Nelma Nunes da Silva', Márcia Aparecida Ferreira de Oliveira² ${ }^{2}$ Mônica Silvia Rodrigues de Oliveira', Heloísa Garcia Claro ${ }^{\mathbf{3}}$, Ivan Filipe de Almeida Lopes Fernandes ${ }^{\mathbf{4}}$, Gabriella de Andrade Boska², Ronédia Monteiro Bosque ${ }^{1}$

DOI: $10.1590 / 0103-1104202012719$

RESUMO Este artigo objetivou analisar comparativamente a satisfação de indivíduos negros e não negros assistidos por Centros de Atenção Psicossocial em Álcool e Outras Drogas de três estados brasileiros. É um estudo quantitativo, transversal e avaliativo, cujos dados foram coletados por meio de entrevistas seguidas de análise inferencial. Participaram do estudo 707 indivíduos, a maioria homens, autodeclarados negros, com média de idade de 44 anos. Os resultados mostraram que indivíduos negros e não negros estavam igualmente satisfeitos com a assistência ofertada, sobretudo com a competência e compreensão dos profissionais, acolhimento e ajuda recebida, o que contradiz a literatura tradicional que desvela racismo nos serviços de saúde. A avalição é a de que os Centros de Atenção Psicossocial em Álcool e Outras Drogas são locais potentes e diferenciados do sistema de saúde, mais acolhedores e inclusivos, para o cuidado de qualquer tipo de população com necessidades decorrentes do uso de substâncias. Esse resultado é coerente com a história desses dispositivos de saúde em nosso país, sendo serviços centrais da rede substitutiva aos cuidados hegemônicos e asilares.

PALAVRAS-CHAVE Serviços de saúde mental. Pesquisa sobre serviços de saúde. Satisfação do paciente. Grupo com ancestrais do continente africano.

1 Universidade Federal do Amapá (Unifap) - Macapá (AP), Brasil.

nelmans@hotmail.com

2 Universidade de São Paulo (USP), Escola de Enfermagem - São Paulo (SP), Brasil.

3 Universidade Estadual de Campinas (Unicamp), Faculdade de Enfermagem (Fenf) - Campinas (SP)

Brasil.

4 Universidade Federal do ABC (UFABC) - Santo André (SP), Brasil.
ABSTRACT The paper aimed to compare the satisfaction of black and non-black individuals assisted by Psychosocial Care Centers for Alcohol and Drugs in three Brazilian states. This is a quantitative, cross-sectional and evaluative study, with inferential analysis of the data we collected through interviews. A total of 707 individuals participated in the study, mostly men, self-declared black, with an average age of 44 years. The results show that black and non-black individuals were equally satisfied with the assistance offered, especially with the competence and understanding of the professionals, embracement and help received. These results contradict the traditional literature that reveals racism in health services. We believe that the Psychosocial Care Centers on Alcohol and Drugs are powerful and differentiated services in the health system, more welcoming and inclusive of their. This result is consistent with the history of these health devices in our country, being central services in the network that is substitutive of hegemonic and asylum-centered psychiatric care.

KEYWORDS Mental health service. Health services research. Patient satisfaction. African continental ancestry group. 


\section{Introdução}

Com o movimento de reforma psiquiátrica brasileira, ocorreu um processo de reestruturação dos serviços de saúde mental com objetivo de promover assistência integral e humanizada. Desde 2002, com a implantação do Centro de Atenção Psicossocial em Álcool e Outras Drogas (Caps AD), iniciou-se a construção de uma rede de serviços para a população que apresenta problemas com o consumo de substâncias psicoativas. O Caps AD utiliza a lógica de redução de danos e tem por objetivo atender às necessidades dos usuários dos serviços de forma sistemática e ativa segundo o ambiente cultural e comunitário em que estão inseridos, de acordo com os princípios da reforma psiquiátrica e da constituição brasileira de igualdade e universalidade ${ }^{1-3}$.

Todavia, esse acesso universal e igualitário à saúde tem esbarrado num conjunto de fatores que incluem as diferenças socioeconômicas existentes, sendo a população negra bastante impactada por motivos que se relacionam com o longo período histórico de escravidão no Brasil, racismo, pobreza, existência de doenças específicas e por constituir mais da metade da população brasileira (53,1\%); são negros (pretos e pardos) autodeclarados, demandando políticas públicas com ações específicas de equilibrar tais diferenças $\mathbf{4}^{\mathbf{5}}$.

Nessa perspectiva, a Política Nacional de Saúde Integral da População Negra (PNSIPN), instituída pelo Ministério da Saúde em 2009 com objetivo de combater a discriminação étnico-racial nos serviços de saúde bem como equidade no acesso à saúde da população negra, inclui estratégias para o fortalecimento da atenção à saúde mental, em especial àqueles com problemas decorrentes do uso de álcool e outras drogas 5 .

Pesquisas recentes têm mostrado que a população negra ainda tem menos acesso à saúde do que a população branca. A população negra relata que, em algum momento, já foi discriminada durante o processo assistencial. Além disso, a maioria das pessoas negras não possui plano de saúde (78,8\%), dependendo, consequentemente, dos serviços do Sistema Único de Saúde (SUS) e representando mais da maioria do seu público total atendido ${ }^{6-8}$.

Nesse sentido, o serviço do Caps AD é fundamentalmente importante para a assistência aos indivíduos negros que fazem uso de álcool e outras drogas, tornando-se necessária sua constante avaliação. Estudo recente sobre o padrão do uso de crack mostrou que a maioria desses indivíduos era negra ${ }^{9}$. Em outro estudo de revisão, foi sugerido que o recorte racial é um importante marcador social, que pode gerar evidências científicas e contribuições para melhor atuação dos serviços de saúde ${ }^{\mathbf{1 0}}$.

Além disso, a satisfação com esses serviços contribui para melhor qualidade de vida, diminuição no número de internações hospitalares, melhor adesão e frequência ao tratamento ${ }^{11}$. Logo, um estudo que analise a satisfação de negros e não negros dos Caps $\mathrm{AD}$ promoveria mais informações e compreensão a respeito do assunto. Desse modo, este estudo tem por objetivo analisar comparativamente, numa perspectiva racial, a satisfação de indivíduos negros e não negros assistidos por Caps $\mathrm{AD}$ de três estados brasileiros.

\section{Material e métodos}

Trata-se de pesquisa avaliativa, transversal de abordagem quantitativa. Participaram do estudo 707 usuários de 30 Caps AD distribuídos em 19 cidades dos três estados selecionados: São Paulo (SP), Minas Gerais (MG) e Amapá (AP). Sortearam-se 30 nomes de possíveis participantes da pesquisa para cada Caps AD. Considerou-se perda mediante recusa ou impossibilidade de contato após cinco tentativas, realizando-se novo sorteio para reposição. O cálculo amostral foi realizado com base em estudo piloto. Incluíram-se indivíduos com idades entre 18 e 65 anos, participantes das atividades do Caps AD há pelo menos seis meses, com problemas decorrentes do uso abusivo e 
da dependência ao álcool e outras drogas, com capacidade cognitiva para responder aos questionários e que concordaram em assinar o Termo de Consentimento Livre e Esclarecido.

A coleta de dados ocorreu de 2013 a 2016. Para avaliar a satisfação com o serviço, utilizou-se a Escala de Avaliação da Satisfação dos Pacientes com os Serviços de Saúde Mental Satis-BR. Essa escala foi elaborada pela Divisão de Saúde Mental da Organização Mundial da Saúde e validada para o Brasil11. Um estudo recente de validação sustenta que a escala possui índice de consistência interna (alfa de Cronbach) igual a 0,88 , valor considerado válido pela literatura. Além disso, a escala apresentou validade convergente evidenciada pela comparação da correlação com escores de escala que mensura constructo semelhante. Análise de correlação dos fatores com a escala global demonstra correlação positiva e estatisticamente significante ${ }^{\mathbf{1 1}}$.

A Satis-BR possui 12 itens agrupados em três fatores para avaliar o grau de satisfação dos pacientes em relação à Competência e compreensão da equipe a respeito do problema do paciente (fator 1); Ajuda recebida e acolhida da equipe (fator 2); e Condições físicas e conforto do serviço (fator 3), incluídos também na escala global. As alternativas de resposta aos itens estão dispostas em uma escala do tipo Likert com 5 pontos, na qual 1 representa que o usuário está muito insatisfeito e 5 que está muito satisfeito com o serviço. $\mathrm{O}$ instrumento foi aplicado utilizando-se formulário on-line do Google Forms ${ }^{\circledR}$.

Os dados coletados foram tabulados, codificados e processados utilizando-se os programas Stata ${ }^{\circledR}$ versão 15 e IBM SPSS versão 20 para Windows ${ }^{\circledR}$.

Uma análise de regressão logística múltipla foi realizada para examinar a relação entre as variáveis independentes com o fato de o usuário ser negro ou não negro, controlando-se a análise para escolaridade, alfabetização e renda. A regressão logística múltipla encontra a equação que melhor prediz o valor das variáveis dependentes para os valores da variável independente, neste caso, negro ou não negro ${ }^{\mathbf{1 2}}$. Em outras palavras, a análise permite visualizar a probabilidade de obter um valor particular para os desfechos acontecerem com negros comparados a não negros. Partiu-se do pressuposto que há desigualdade no tratamento dos negros em comparação aos não negros, confirmado pela literatura, uma vez que estudos internacionais e nacionais mostram que o racismo reforça até mesmo a distribuição de recursos na área da saúde, o que se configura obstáculo para saúde integral, universal e equânime ${ }^{\text {13-17 }}$.

Nesta análise inferencial da desigualdade no tratamento, controlamos as variáveis para renda, alfabetização e escolaridade, para que não se confunda o efeito da variável de raça com esses determinantes sociais. Dessa forma, quando encontrada associação da variável de raça nas variáveis dependentes, pode-se afirmar que esse efeito é independente da alfabetização, escolaridade e renda dos indivíduos. Consideram-se estatisticamente significante os valores de $\mathrm{p}<=0,05$.

$\mathrm{O}$ estudo deriva de pesquisa matricial de avaliação dos Caps $\mathrm{AD}$, aprovado pelo Comitê de Ética em Pesquisa da Escola de Enfermagem da Universidade de São Paulo e da Secretaria Municipal de Saúde de São Paulo sob os pareceres 1001/2011 e 054/12, respectivamente, considerando os preceitos éticos da Resolução $n^{\circ}$ 196/96 e no 466/12 do Conselho Nacional de Ética em Pesquisa.

\section{Resultados}

Do total de 707 participantes do estudo, 420 (59,4\%) eram negros, dos quais 297 (42\%) pretos e 123 (17,4\%) pardos, e $287(40,6)$ eram não negros. Seu perfil está descrito na distribuição de frequências das variáveis sociodemográficas segundo estado, sexo, etnicidade, estado civil, renda familiar, alfabetização, escolaridade e idade (tabela 1). 
Tabela 1. Distribuição das frequências de variáveis sociodemográficas de usuários dos Caps AD. Amapá-AP, Minas Gerais-MG, São Paulo-SP, Brasil

\begin{tabular}{|c|c|c|}
\hline Variáveis & Frequência (n) & Percentual \% \\
\hline \multicolumn{3}{|l|}{ Estado } \\
\hline Amapá & 60 & 8,5 \\
\hline Minas Gerais & 330 & 46,7 \\
\hline São Paulo & 317 & 44,8 \\
\hline \multicolumn{3}{|l|}{ Sexo } \\
\hline Feminino & 302 & 42,7 \\
\hline Masculino & 405 & 57,3 \\
\hline \multicolumn{3}{|l|}{ Etnicidade } \\
\hline Não-negros & 287 & 40,6 \\
\hline Negros (Preto/Pardo) & $420(297 / 123)$ & $59,4(42 / 17,4)$ \\
\hline \multicolumn{3}{|l|}{ Estado civil } \\
\hline Casado & 213 & 30,1 \\
\hline Divorciado ou separado & 67 & 9,4 \\
\hline Solteiro & 417 & 59 \\
\hline Viúvo & 10 & 1,4 \\
\hline \multicolumn{3}{|l|}{ Renda Familiar } \\
\hline Até 2 salários mínimos & 503 & 71,1 \\
\hline De 2 a 4 salários mínimos & 148 & 20,9 \\
\hline Acima de 4 salários mínimos & 56 & 7,9 \\
\hline \multicolumn{3}{|l|}{ Sabe ler } \\
\hline Não & 40 & 5,7 \\
\hline Sim & 667 & 94,3 \\
\hline \multicolumn{3}{|l|}{ Escolaridade } \\
\hline Sem escolaridade & 18 & 2,5 \\
\hline Ensino fundamental incompleto & 274 & 38,8 \\
\hline Ensino fundamental completo & 96 & 13,6 \\
\hline Ensino médio incompleto & 86 & 12,2 \\
\hline Ensino médio completo & 134 & 19 \\
\hline Ensino técnico incompleto & 3 & 0,4 \\
\hline Ensino técnico completo & 12 & 1,7 \\
\hline Ensino superior incompleto & 37 & 5,2 \\
\hline Ensino superior completo & 31 & 4,4 \\
\hline Pós-graduação incompleta & 1 & 0,1 \\
\hline Pós-graduação completa & 4 & 0,6 \\
\hline Não informado & 11 & 1,6 \\
\hline \multicolumn{3}{|l|}{ Idade } \\
\hline Média & 44 & \\
\hline Mediana & 44 & \\
\hline Mínimo & 18 & \\
\hline Máximo & 76 & \\
\hline
\end{tabular}

Fonte: Elaboração própria.

Caps AD: Centro de Atenção Psicossocial em Álcool e Outras Drogas. 
A tabela 2 apresenta a distribuição comparativa da frequência percentual de variáveis sociodemográficas e econômicas de negros e não negros. Observa-se que a renda familiar da maioria dos negros $(75,2 \%)$ é de até 2 salários mínimos, enquanto para não negros o percentual cai para $65,2 \%$. Também se observa que apenas 5,5\% dos negros recebem acima de 4 salários mínimos, enquanto para não negros este percentual sobe para $11,4 \%$.

Sobre a escolaridade, apesar de a maioria dos negros (93,3\%) e não negros (95,8\%) saber ler, observa-se um maior grau de instrução entre os não negros, pois, tanto no ensino fundamental completo como no médio completo e superior completo, apresentam maiores percentuais comparado aos negros, sendo que estes apresentam ainda maior porcentagem sem escolaridade (33,1\%) comparado aos não negros (18,1\%).

Quanto ao tempo em que os participantes são assistidos nos serviços, destacamos que há maior proporção de indivíduos negros que frequentam os serviços há menos tempo.

Tabela 2. Distribuição da frequência percentual de variáveis sociodemográficas de negros e não negros dos Caps AD. Amapá-AP, Minas Gerais-MG, São Paulo-SP, Brasil

\begin{tabular}{|c|c|c|}
\hline Variáveis & Não negro Percentual \% & Negro Percentual \% \\
\hline \multicolumn{3}{|l|}{ Sabe ler } \\
\hline Não & 4,2 & 6,7 \\
\hline Sim & 95,8 & 93,3 \\
\hline \multicolumn{3}{|l|}{ Escolaridade } \\
\hline Sem escolaridade & 18,1 & 33,1 \\
\hline Ensino fundamental incompleto & 25,1 & 30,0 \\
\hline Ensino fundamental completo & 16,4 & 12,1 \\
\hline Ensino médio incompleto & 8,0 & 6,0 \\
\hline Ensino médio completo & 13,2 & 7,1 \\
\hline Ensino técnico incompleto & 1,7 & 2,1 \\
\hline Ensino técnico completo & 4,5 & 4,5 \\
\hline Ensino superior incompleto & 5,6 & 3,1 \\
\hline Ensino superior completo & 5,9 & 1,4 \\
\hline Pós-graduação incompleta & 0,3 & 0,0 \\
\hline Pós-graduação completa & 1,0 & 0,2 \\
\hline Não informado & 0,0 & 0,2 \\
\hline \multicolumn{3}{|l|}{ Renda Familiar no último mês } \\
\hline Até 2 salários mínimos & 65,2 & 75,2 \\
\hline De 2 a 4 salários mínimos & 23,4 & 19,3 \\
\hline Acima de 4 salários mínimos & 11,4 & 5,5 \\
\hline \multicolumn{3}{|l|}{ Tempo de frequência no serviço } \\
\hline 6 meses & 8,4 & 15,5 \\
\hline 7 a 12 meses & 22,3 & 21 \\
\hline 1 a 2 anos & 24,7 & 22,1 \\
\hline 3 a 5 anos & 28,2 & 26,4 \\
\hline Mais de 5 anos & 16,4 & 15 \\
\hline
\end{tabular}

Fonte: Elaboração própria.

Caps AD: Centro de Atenção Psicossocial em Álcool e Outras Drogas. 
A tabela 3 apresenta os resultados da análise descritiva dos escores de satisfação global e por fatores $(1,2,3,4)$ dos participantes para cada item da escala Satis-BR, em valores de mediana. Os resultados mostram uma avaliação positiva da satisfação global em todos os itens, com mediana de 4,4 para negros e não negros.

As questões sobre Ajuda recebida e Acolhida (fator 2) receberam melhor avaliação, com valores de 4,6 pontos para negros e não negros. O mesmo se deu com o fator 1 , sobre a Competência e Compreensão da equipe, que apresentou pontuações positivas de 4,5 e 4,4 para negros e não negros, respectivamente.

Apesar de apresentar um bom escore, as condições físicas e conforto do Caps AD (fator 3) apresentaram a menor pontuação dos itens da escala, com escore de 4,0 pontos para ambos os grupos.

O fator 4 - Satisfat 4 comparou os resultados obtidos na Satis-BR com os dados da CSQ-8 (Client Satisfaction Questionarie) - escala padronizada de avaliação da satisfação com os serviços de saúde mental, desenvolvida por Larsen, Attkisson, Hargreaves e Nguyen (1979). Essa comparação entre as respostas das escalas visa a reavaliar constantemente a validade concomitante da escala utilizada no estudo. Esse fator também obteve resultado positivo, com valores de 4,6 para negros e 4,5 para não negros.

Tabela 3. Medidas descritivas dos Escores da Escala Satis-BR dos usuários negros e não negros de Caps AD. Amapá-AP, Minas Gerais-MG, São Paulo-SP, Brasil

\begin{tabular}{lrr}
\hline Fator & Negros (Mediana) & Não Negro (Mediana) \\
\hline Satisglobal & 4,4 & 4,4 \\
Fator 1: Competência e Compreensão & 4,5 & 4,4 \\
Fator 2: Ajuda recebida e Acolhida & 4,6 & 4,6 \\
Fator 3: Condições físicas e conforto & 4,0 & 4,0 \\
Fator 4: Satis-BR x CSQ-8 & 4,6 & 4,5 \\
\hline
\end{tabular}

Fonte: Elaboração própria.

Caps AD: Centro de Atenção Psicossocial em Álcool e Outras Drogas; Satis-BR: Escala de Satisfação; Satisglobal: satisfação global; CSQ

Client Satisfaction Questionnarie.

A tabela 4 apresenta a análise múltipla de regressão logística da avaliação da satisfação entre os participantes negros e não negros. Os resultados obtidos demonstram que não houve associações significativas para este estudo, o fato do usuário ser negro, mesmo controlando a influência das variáveis alfabetização, escolaridade e renda.

Tabela 4. Regressão logística da avaliação da satisfação entre os usuários negros e não negros de Caps AD. AP, MG, SP, Brasil

\begin{tabular}{lcccrr}
\hline Regressão Logística & Satisglobal & Fator 1 & Fator 2 & Fator 3 & Fator 4 \\
\hline Negro & & & & & \\
\hline & $-0,044$ & $-0,013$ & $-0,06$ & $-0,126$ & $-0,057$ \\
& 0,038 & 0,042 & 0,043 & $0,067^{\star}$ & 0,047 \\
\hline Alfabetização & & & & & \\
\hline & $-0,135$ & $-0,115$ & $-0,101$ & $-0,258$ & $-0,089$ \\
& $0,082^{*}$ & 0,089 & 0,092 & 0,142 & 0,101 \\
\hline
\end{tabular}


Tabela 4. (cont.)

\begin{tabular}{|c|c|c|c|c|c|}
\hline Regressão Logística & Satisglobal & Fator 1 & Fator 2 & Fator 3 & Fator 4 \\
\hline \multicolumn{6}{|l|}{ Escolaridade } \\
\hline \multirow[t]{2}{*}{ Fundamental Incompleto } & $-0,004$ & 0,028 & $-0,059$ & $-0,037$ & $-0,007$ \\
\hline & 0,05 & 0,055 & 0,057 & 0,087 & 0,062 \\
\hline \multirow[t]{2}{*}{ Fundamental Completo } & $-0,064$ & $-0,034$ & $-0,035$ & $-0,215$ & $-0,025$ \\
\hline & 0,062 & 0,068 & 0,07 & $0,108^{\star \star}$ & 0,077 \\
\hline \multirow[t]{2}{*}{ Médio Incompleto } & $-0,001$ & 0,088 & $-0,105$ & $-0,158$ & 0,003 \\
\hline & 0,081 & 0,088 & 0,091 & 0,14 & 0,099 \\
\hline \multirow[t]{2}{*}{ Médio Completo } & 0,125 & 0,115 & 0,03 & 0,302 & 0,043 \\
\hline & 0,071 & 0,077 & 0,08 & $0,123^{\star \star}$ & 0,087 \\
\hline \multirow[t]{2}{*}{ Técnico Incompleto } & $-0,096$ & $-0,01$ & $-0,212$ & $-0,221$ & $-0,092$ \\
\hline & 0,135 & 0,147 & 0,153 & 0,235 & 0,167 \\
\hline \multirow[t]{2}{*}{ Técnico Completo } & $-0,235$ & $-0,179$ & $-0,297$ & $-0,338$ & $-0,168$ \\
\hline & $0,094^{\star \star}$ & 0,102 & $0,106^{\star \star \star}$ & $0,163^{\star \star}$ & 0,116 \\
\hline \multirow[t]{2}{*}{ Superior } & $-0,135$ & $-0,048$ & $-0,241$ & $-0,283$ & $-0,137$ \\
\hline & 0,083 & 0,09 & $0,093^{\star \star}$ & $0,144^{\star \star}$ & 0,102 \\
\hline \multicolumn{6}{|l|}{ Renda Familiar } \\
\hline \multirow[t]{2}{*}{2 a 4 salários mínimos } & 0,134 & 0,109 & 0,157 & 0,185 & 0,161 \\
\hline & $0,047^{\star \star}$ & $0,051^{\star \star}$ & $0,053^{\star \star *}$ & $0,082^{\star \star}$ & $0,058^{\star \star *}$ \\
\hline \multirow[t]{2}{*}{ Acima de 4 salários mínimos } & $-0,007$ & 0,02 & $-0,023$ & $-0,078$ & 0,016 \\
\hline & 0,075 & 0,082 & 0,085 & 0,131 & 0,093 \\
\hline \multirow[t]{2}{*}{-cons } & 4,493 & 4,476 & 4,7 & 4,24 & 4,538 \\
\hline & $0,083^{\star \star \star}$ & $0,091^{\star \star \star}$ & $0,094^{\star \star \star}$ & $0,145^{\star \star \star}$ & $0,103^{\star \star \star}$ \\
\hline F statistic & 2,6 & 1,4 & 2,5 & 3,4 & 1,3 \\
\hline Adjusted R-squared & 0,02 & 0,01 & 0,02 & 0,04 & 0 \\
\hline $\mathrm{N}$ & 707 & 707 & 707 & 707 & 707 \\
\hline
\end{tabular}

Fonte: Elaboração própria.

Caps AD: Centro de Atenção Psicossocial em Álcool e Outras Drogas; AP=Amapá; MG=Minas Gerais; SP=São Paulo; ' ${ }^{*}<0,1,{ }^{\star *} p<0,05$, ${ }^{\star \star *} p<0,01$.

\section{Discussão}

Participaram deste estudo 707 indivíduos dos Caps AD de três estados brasileiros: SP, MG e AP, com as seguintes características sociodemográficas: a maioria eram homens $(57,3 \%)$, solteiros (59,0\%) e com média de idade de 44 anos. Dados semelhantes a outros estudos da área9.

Desse total, 420 eram negros (297 pretos e 123 pardos) e $287(40,6)$ eram não negros. Comparativamente, o número de negros $(75,2 \%)$ que possuem renda familiar de até
2 salários mínimos é maior do que o de não negros $(65,2 \%)$. E, apesar da maioria dos negros e não negros saber ler, observou-se maior grau de instrução entre os não negros. Tais diferenças nas variáveis sociodemográficas têm sido associadas na literatura internacional ao pertencimento racial que leva a inadequações quanto à condição e estilo de vida, empregabilidade, acesso à alimentação e aos serviços de saúde ${ }^{\mathbf{1 8}, 19}$.

Em seguida, foi realizada avaliação da satisfação frente à assistência recebida por 
meio da aplicação da Escala Satis-BR com recorte racial, sob o controle das variáveis alfabetização, escolaridade e renda. Partiu-se do pressuposto que há diferença na assistência oferecida entre negros e não negros, com consequente impacto na satisfação referida.

Nesse caso, a análise de regressão logística múltipla mostrou que não houve diferenças na satisfação global entre os dois grupos populacionais, conferindo uma boa avaliação geral com a assistência prestada pelos Caps AD estudados, sendo esse efeito independente da alfabetização, escolaridade e renda desses indivíduos.

Estudos têm reafirmado que, embora a população negra apresente as mesmas doenças da população em geral, comparativamente, a maneira como discorrem e como o cuidado é ofertado é diferente. Tais diferenças se relacionam com situações de preconceito, estigmatização, exclusão social e desigualdades, com consequente acesso restrito aos serviços de saúde. Neste último, indicando interferências quanto ao relacionamento profissional nos serviços ${ }^{20,21}$.

É importante considerar as singularidades da população negra em diversos cenários e $\operatorname{contextos}^{22}$, sendo necessário refletir sobre como os resultados deste estudo se justificam diante dos níveis semelhantes de satisfação analisados para os grupos de negros e de não negros conferidos aos serviços dos Caps AD, na perspectiva racial.

Os avanços da reforma psiquiátrica brasileira trouxeram importante expansão no número de Caps $\mathrm{AD}$ em todo o território nacional. Embora esse número ainda seja insuficiente para o atendimento das demandas da população, a literatura tem mostrado que tais dispositivos possuem papel fundamental na rede de atenção psicossocial como protagonistas no processo de cuidar as pessoas com necessidades decorrentes do uso de álcool e outras drogas 23 .

Aliado a isso e apesar de incipiente, houve nos últimos anos um aumento de pesquisas brasileiras que abordam questões étnico-raciais, o que contribui para melhor compreensão e acesso às ações e serviços de saúde, bem como de políticas públicas específicas ${ }^{22}$, incluindo a saúde da população negra, avaliação da rede de serviços, e processos de trabalho, entre outros.

A literatura nacional e internacional sobre o processo de avaliação em saúde mental tem identificado que a satisfação positiva do usuário com o serviço se relaciona aos vínculos formados com a equipe de saúde, acolhimento nas consultas, qualidade dos serviços, acompanhamento durante e após o tratamento e garantia de acesso ${ }^{\mathbf{1 7} 23-26}$, dado que, pode ser reforçado neste presente estudo, ao não observarmos diferenças relacionadas a raça ou cor.

Por outro lado, essa satisfação positiva com o serviço também tem sido relacionada a um certo temor pelos participantes de possível perda de direito de acesso ao cuidado, muitas vezes expressado em avaliações negativas mas não necessariamente expressado nas opiniões, numa situação conhecida como viés de gratidão ${ }^{27}$. É preciso considerar que o Caps atende a demandas da população bastante fragilizada do ponto de vista físico, emocional e social, que são agravadas pelo estigma, discriminação e preconceito que envolve o consumo de substâncias e, além disso, são extremamente intensificadas por questões raciais, sendo essencial o acolhimento de todos que acessam os serviços ${ }^{\mathbf{6}, 28}$. Fatores relevantes que poderiam influenciar nos achados deste estudo.

A satisfação expressada pelos indivíduos assistidos pelos serviços de saúde mental envolve a necessidade de mudança fundamental no contexto da relação do usuário com os profissionais de saúde para facilitação do engajamento no cuidado oferecido ${ }^{25}$, em conjunto com oferta assistencial sem interferência de julgamentos e independentemente de sua raça ou cor ${ }^{29,30}$, como também demonstrado por este estudo.

Quanto aos fatores da escala Satis-BR avaliados nesta pesquisa, constatou-se que $o$ fator 1 sobre Competência e Compreensão da Equipe e o fator 2, sobre Ajuda e Acolhimento, receberam as melhores avaliações, corroborando outros estudos desta área ${ }^{17,31}$. Já o fator 3, relacionado à Estrutura física e Conforto 
do serviço, apesar de obter avaliação positiva, recebeu pontuação mais baixa do que os fatores $1 \mathrm{e} 2$ por ambos os grupos populacionais estudados. Estudo recente obteve resultado semelhante, mostrando boa avaliação do Caps $\mathrm{AD}$ por $88,3 \%$ dos indivíduos, em sua maioria negros, que relataram que o serviço poderia ser melhorado quanto à estrutura física, indicando que essa possa ser a maior debilidade encontrada ${ }^{17}$, debilidade esta, também revelada em outros estudos ${ }^{32,33}$.

Avaliamos que os resultados positivos observados pelos indivíduos assistidos nos serviços, independentemente de sua raça ou cor, indicam que os Caps $\mathrm{AD}$ são serviços que superam a construção rígida e segregacionista de outros modelos de cuidado em saúde no Brasil e no mundo ${ }^{13-16}$, se amparando em sua característica progressista desde sua implantação.

Os Caps são vistos pela literatura como serviços que dão espaço à diversidade, cidadania, valorização cultural, com foco nos direitos e protagonismo dos indivíduos assistidos ${ }^{34}$. Compreendemos que o papel que os Caps AD ocupam na Reforma Psiquiátrica, sua história e serviço central por um sistema de saúde integral são responsáveis pelos resultados aqui encontrados.

Infelizmente, o cenário da integralidade do cuidado em saúde mental vem sendo ameaçado nos últimos anos por práticas e serviços que fazem emergir novamente o pensamento conservador e asilar. Alguns serviços, como as comunidades terapêuticas, utilizam de laborterapia e oferecem cura por meio da religião, afastando-se completamente das práticas baseadas em evidências ${ }^{35}$. Essas práticas prejudicam os avanços da reforma psiquiátrica brasileira, e avaliamos pertinente a literatura que marca tais práticas como retrocesso, o que pode em breve ameaçar a existência da rede de atenção psicossocial, alinhando-se novamente com perspectivas manicomiais e asilares ${ }^{35}$.

Todavia, nos deparamos com a dialética entre práticas conservadoras e progressistas, que provocam tensões no cuidado no campo psicossocial valorizado com a reforma psiquiátrica brasileira entre os anos 1980 e 2016, quando há uma clara onda conservadora no Brasil ${ }^{36,37}$, que se reflete diretamente no sistema como um todo. Os embates entre cuidado asilar e o cuidado na comunidade, o campo psiquiátrico versus o campo psicossocial, o objetivo de abstinência total como norma e a redução de danos como opção de escolha e, principalmente, a política de guerra às drogas historicamente instituída no País contra a população pobre, negra e periférica desafiam as práticas em saúde mental e em álcool e drogas e impõem constantes barreiras a serem ultrapassadas. É necessário apoiar-nos nas evidências científicas e no cuidado humanizado, inclusivo e integral, como o produzido pelos Caps AD, para resistir a esse cenário de retrocesso e repensar políticas públicas que sustentem o modelo de descriminalização do sujeito ${ }^{35}$.

A assistência prestada pelos Caps $\mathrm{AD}$ dos estados de São Paulo, Minas Gerais e Amapá é satisfatória, independentemente da variável raça e cor, com destaque para a competência e acolhimento da equipe de saúde, dado que evidencia a potencialidade dos serviços comunitários de saúde mental para atender toda a população com necessidades decorrentes do uso de substâncias, sem reforçar o local de diferença vivido tanto pelo consumo de drogas como pela questão étnico-racial.

\section{Limitações do estudo}

Por se tratar de estudo transversal, este estudo não avaliou as relações de causa e efeito. Além disso, a incipiência de publicações sobre o tema na literatura restringiu a discussão de alguns achados.

\section{Contribuições do estudo para a prática}

Considera-se os Caps AD como satisfatórios entre pessoas que consomem álcool e outras drogas, independentemente de raça ou cor. Avaliar a satisfação com o serviço mediante 
recorte racial permite dar voz aos participantes, especialmente da população negra, refletindo sobre o papel e qualidade da assistência prestada. Tal atitude propicia a contínua compreensão, elucidação e corresponsabilização dos atores envolvidos, i.e., profissionais, gestores, sociedade e assistidos pelo serviço, em direção à assistência cada vez mais equânime, integral, eficaz, participativa, não julgadora ou estigmatizante.

\section{Conclusões}

Os achados deste estudo mostraram, em geral, boa satisfação frente ao serviço oferecido nos Caps AD analisados, conferindo possibilidade de maior acesso ao tratamento, bem como visibilidade positiva desses dispositivos como locais potentes para o cuidado na rede. Embora não tenha havido diferença no nível de satisfação referida entre negros e não negros, tal semelhança pode ser um indicativo de qualidade do atendimento.

É certo que pesquisas de avaliação desses serviços são necessárias à medida que possibilitam reflexões sobre o papel e qualidade da assistência prestada, além de dar voz aos usuários do serviço sobre as experiências vividas nesses locais. Ademais, a avaliação mediante o recorte racial contribui para o fortalecimento da Política Nacional de Saúde Integral da População Negra (PNSIPN) voltada à atenção à saúde mental, permitindo, assim, maior compreensão em direção à assistência cada vez mais equitativa e integral.

As melhores pontuações, obtidas tanto por negros quanto não negros, estiveram relacionadas aos fatores ajuda, acolhimento e compreensão, relacionados ao vínculo entre profissional e usuário. Tais fatores indicam, respectivamente, o cumprimento dos objetivos do serviço e sua importância, representando importante avanço da reforma psiquiátrica brasileira na perspectiva de atendimento e acolhimento a todo tipo de população, apesar de dificuldades enfrentadas quanto ao conforto e estrutura física, fator com menor pontuação de satisfação comparado aos demais fatores.

Diante da incipiência de publicações que abordam esta temática sob a perspectiva racial, este estudo representa uma estimativa importante da avaliação da população negra desses três estados, sendo necessária ampliação da avaliação para outras regiões do País. Desse modo, espera-se que os resultados contribuam para as políticas locais no aprimoramento de ações que ampliem a atenção integral, gerando maiores evidências com recorte racial.

\section{Colaboradores}

Silva NN (0000-0003-2790-1792)* contribuiu para concepção, planejamento, análise e interpretação dos dados, e revisão crítica do conteúdo. Oliveira MAF (0000-0002-10698700)* contribuiu para concepção e planejamento, interpretação dos dados e revisão crítica do conteúdo. Oliveira MSR (00000002-2632-3639)* contribuiu para revisão crítica e aprovação final do conteúdo. Claro HG (0000-0003-1504-7074)* contribuiu para análise e interpretação dos dados e revisão crítica. Fernandes IFAL (0000-0002-42364393)* contribuiu para análise dos dados. Boska GA (0000-0002-5827-6486)* contribuiu para revisão e aprovação final. Bosque RM (0000-0002-4445-3250)* contribuiu para concepção e planejamento do manuscrito. 


\section{Referências}

1. Fagundes Júnior HM, Desviat M, Silva PRF. Reforma Psiquiátrica no Rio de Janeiro: situação atual e perspectivas futuras. Ciênc. Saúde Colet. [internet]. 2016 [acesso em 2017 nov 17]; 21(5):1449-60. Disponível em: http://www.scielo.br/pdf/csc/v2ln5/14138123-csc-21-05-1449.pdf.

2. Brasil. Ministério da Saúde. Portaria GM no 336, de 19 de fevereiro de 2002. Estabelece sobre a proteção e os direitos das pessoas portadoras de doença mental e redireciona o modelo assistencial em saúde mental. Diário Oficial da União. 19 Fev 2002. [acesso em 2020 set 1]. Disponível em: https://bvsms.saude.gov. br/bvs/saudelegis/gm/2002/prt0336_19_02_2002. htm.

3. Brasil. Ministério da Saúde. Portaria n ${ }^{\circ} 130$, de 26 de janeiro de 2012. Redefine o Centro de Atenção Psicossocial de Álcool e outras Drogas 24 h (CAPSad III) e os respectivos incentivos financeiros. Diário Oficial da União. 26 Jan 2012. [acesso em 2020 set 1]. Disponível em: http://www.saude.gov.br/images/pdf/2015/ marco/10/PORTARIA-130-26--JANEIRO-2012.pdf.

4. Jaccoud L. O combate ao racismo e à desigualdade: $\mathrm{O}$ desafio das políticas públicas de promoção à saúde. In: Theodoro M, Jaccoud LB, Osório GR, et al., organizadores. As políticas públicas e a desigualdade racial no Brasil: 120 anos após a abolição [internet]. Brasília, DF: IPEA; 2008 [acesso em 2019 jul 23]. 131 p. Disponível em: http://www.clam.org.br/bibliotecadigital/uploads/publicacoes/1107_1899_Livrodesigualdadesraciais.pdf.

5. Brasil. Ministério da Saúde, Secretaria de Gestão Estratégica e Participativa. Painel de Indicadores do SUS $n^{\circ}$ 10. Temático Saúde da População Negra. [internet]. Brasília, DF: Ministério da Saúde; 2016 [acesso em 2019 jul 24]; 83 p. Disponível em: http://bvsms. saude.gov.br/bvs/publicacoes/tematico_saude_populacao_negra_v._7.pdf.

6. Instituto Brasileiro de Geografia e Estatística. Pesquisa Nacional de Saúde 2013[internet]. Rio de Janeiro: IBGE; 2013 [acesso em 2019 jul 30]; 105 p. Disponí- vel em: https://biblioteca.ibge.gov.br/visualizacao/ livros/liv94074.pdf.

7. Brasil. Ministério de Saúde. Boletim Epidemiológico. Indicadores de Vigilância em Saúde, analisados segundo a variável raça/cor [internet]. Brasília, DF: Ministério de Saúde, Secretaria de Vigilância em Saúde; 2015 [acesso em 2019 jul 30]; 46(10):35. Disponível em: http://portalarquivos2.saude.gov.br/images/ pdf/2015/abril/22/Boletim-raca-cor-09-04-15-v2.pdf.

8. Instituto Brasileiro de Geografia e Estatística. Síntese de indicadores sociais: uma análise das condições de vida da população brasileira [internet]. Rio de Janeiro: IBGE; 2016. [acesso em 2019 jul 30]. Disponível em: https://biblioteca.ibge.gov.br/visualizacao/ livros/liv98965.pdf.

9. Almeida CS, Luis MAV. Características sociodemográficas e padrão de uso de crack e outras drogas em um CAPS AD. Rev enferm UFPE [internet]. 2017. [acesso em 2019 jul 23]; 11(supl4):1716-1723. Disponível em: https://periodicos.ufpe.br/revistas/revistaenfermagem/article/view/15269/18073.

10. Silva NG, Barros S, Azevedo FC, et al. O quesito raça/ cor nos estudos de caracterização de usuários de Centro de Atenção Psicossocial. Saúde Soc [internet]. 2017 [acesso em 2019 jul 24]; 26(1):100-114. Disponível em: http://www.scielo.br/pdf/sausoc/v26nl/1984-0470-sausoc-26-01-00100.pdf.

11. Bandeira M, Silva MA. Escala de Satisfação dos Pacientes com os Serviços de Saúde Mental (SATIS-BR): estudo de validação. J Bras Psiquiatr [internet]. 2012 [acesso em 2019 jul 24]; 61(3):124-32. Disponível em: http://www.scielo.br/pdf/jbpsiq/v61n3/02.pdf.

12. Marconi MA, Lakatos EM. Fundamentos de metodologia científica [internet]. São Paulo: Atlas; 2003. [acesso em 2019 jul 24]. Disponível em: https://docente.ifrn.edu.br/olivianeta/disciplinas/copy_of_historia-i/historia-ii/china-e-india. 
13. Bailey ZD, Krieger N, Agénor M, et al. Structural racism and health inequities in the USA: evidence and interventions. Lancet. 2017 [acesso em 2020 set 1]; 389(10077):1453-63. Disponível em: https://www. thelancet.com/journals/lancet/article/PIISO140-6736(17)30569-X/fulltext.

14. Pirtle WNL. Racial capitalism: a fundamental cause of novel coronavirus (COVID-19) pandemic inequities in the United States. Health Educ Behav. 2020 [acesso em 2020 abr 26]; 47(4):504-508. Disponível em: https://pubmed.ncbi.nlm.nih.gov/32338071/.

15. Mota CS, Atkin K, Trad LA, et al. Social disparities producing health inequities and shaping sickle cell disorder in Brazil. Heal Sociol Review. 2017 [acesso em 2020 set 1]; 26(3):280-92. Disponível em: https:// www.tandfonline.com/doi/full/10.1080/14461242.2 017.1361855? scroll=top\&needAccess=true.

16. Mayer-Foulkes D, Larrea C. Racial and Ethnic Health Inequities: Bolivia, Brazil, Guatemala and Peru. 2005. [acesso em 2020 set 1]. Disponível em: https:// www.academia.edu/404293/Racial_and_Ethnic_Health_Inequities_Bolivia_Brazil_Guatemala_and_Peru.

17. Bosque RM, Oliveira MAF, Silva NN, et al. Satisfação dos Trabalhadores, Usuários e Familiares dos Centros de Atenção Psicossocial em Álcool e Outras Drogas. Rev enferm UFPE [internet]. 2017 [acesso em 2019 jul 30]; 11(supl11):4598-606. Disponível em: https:// periodicos.ufpe.br/revistas/revistaenfermagem/article/download/231199/25195.

18. United States of America. National Center for Health Statistics. Healthy people 2010: final review. Washington, D.C.: U.S. Government Printing Office Washington; 2012 [acesso em 2019 dez 10]. Disponível em: https://www.cdc.gov/nchs/data/hpdata2010/ hp2010_final_review.pdf.

19. Andresen EM, Miller DK. The future (history) of socioeconomic measurement and implications for improving health outcomes among African Americans. Journals Gerontol Ser A Biol Sci Med Sci. [internet] 2005. [acesso em 2019 dez 10]; 60(10):1345-50. Disponível em: https://doi.org/10.1093/gerona/60.10.1345.
20. Araújo EM, Costa MCN, Hogan VK, et al. A utilização da variável raça/cor em Saúde Pública: possibilidades e limites. Interface [internet]. 2009 [acesso em 2019 dez 10]; 13(31):383-394. Disponível em: http:// dx.doi.org/10.1590/S1414-32832009000400012.

21. Braveman P, Egerter S, Williams DR. The social determinants of health: coming of age. Annu Rev Public Health [internet]. 2011 [acesso em $2019 \mathrm{dez}$ 10]; 32:381-98. Disponível em: https://doi.org/10.1146/ annurev-publhealth-031210-101218.

22. Spricigo JS, Alencastre MB. O Enfermeiro de Unidade Básica de Saúde e o Usuário de Drogas - um estudo em Biguaçu-SC. Rev Lat-Am Enfermagem [internet]. 2004 [acesso em 2019 jul 24]; 12(esp):427-32. Disponível em: http://www.scielo.br/pdf/rlae/v12nspe/v12nspea19.pdf.

23. Brasil. Ministério da Saúde. Sala de Apoio à Gestão Estratégica [internet]. 2016 [acesso em 2019 dez 10]. Disponível em: http://sage.saude.gov.br/.

24. Oliveira MAF, Cestari TY, Pereira MO, et al. Processos de avaliação de serviços de saúde mental: uma revisão integrativa [internet]. Saúde debate. 2014 [acesso em 2019 ago 2]; 38(101):368-378. Disponível em: http://www.scielo.br/pdf/sdeb/v38n101/0103-1104sdeb-38-101-0368.pdf.

25. Newman D, O'Reilly P, Lee SH, et al. Mental health service users' experiences of mental health care: an integrative literature review. J Psychiatr Ment HLT. 2015 [acesso em 2020 set 1]; 22(3):171-82. Disponível em: https://doi.org/10.1111/jpm.12202.

26. Sohn M, Barret H, Talbert J. Predctors of Consumer Satisfaction in Community Mental Health Center Services. Community Ment Health J. 2014; 50(8):922925.

27. Barbosa GC, Oliveira MAF, Moreno V, et al. Satisfação de usuários num Centro de Atenção Psicossocial em Álcool e Outras Drogas. Revista Portuguesa de Enfermagem de Saúde Mental [internet]. 2015 [acesso em 2019 jul 24]; 14(dez2015):31-7. Disponível em: http:// www.scielo.mec.pt/pdf/rpesm/n14/n14a05.pdf. 
28. Porto MSG. Polícia e violência: representações sociais de elites policiais do Distrito Federal. São Paulo em Perspectiva [internet]. 2004 [acesso em 2019jul 24]; 18(132):132-41. Disponível em: http://www.scielo.br/ pdf/spp/v18nl/22235.pdf.

29. Pereira MO, Souza JM, Costa AM, et al. Profile of users of mental health services in the city of Lorena - São Paulo. Acta Paul Enferm [internet]. 2012 [acesso em 2019 jul 24]; 25(11):48-54. Disponível em: http:// www.scielo.br/pdf/ape/v25nl/en_v25nla09.pdf.

30. Lopes F. Experiências desiguais ao nascer, viver, adoecer e morrer: tópicos em saúde da população negra no Brasil. In: Brasil. Ação Nacional de Saúde. FundaSaúde da população negra no Brasil: contribuições para a promoção da equidade [internet]. Brasília, DF: Funasa; 2005. 446 p. [acesso em 2019 jul 24]. Disponível em: http://bvsms.saude.gov.br/bvs/pop_ negra/pdf/saudepopneg.pdf.

31. Resende KIDS, Bandeira M, Oliveira DCR. Assessment of patient, family and staff satisfaction in a mental health service. Paidéia [internet]. 2016 [acesso em 2019 dez 10]. 26(64):245-53. Disponível em: http:// dx.doi.org/10.1590/1982- 43272664201612 .

32. Kantorski LP, Jardim VR, Wetzel C, et al. Satisfação dos usuários dos centros de atenção psicossocial da região Sul do Brasil [internet]. Rev. Saúde Públ. 2009 [acesso em 2019 ago 2]; 43(supl1):29-35. Disponível em: http://www.scielo.br/scielo.php?script=sci_artt ext\&pid=S0034-89102009000800006.
33. Silva MA, Bandeira M, Scalon JD, et al. Satisfação dos pacientes com os serviços de saúde mental: A percepção de mudanças como preditora [internet]. J. bras. Psiquiatr. 2012 [acesso em 2019 ago 2]; 61(2):6471. Disponível em: http://www.scielo.br/pdf/jbpsiq/ v6ln2/02.pdf.

34. Lavrador MCC, Lima Ribeiro W. As forças do CAPS: uma experiência cartográfica. Rev. Psicol. Saúde. 2015 [acesso em 2020 set 1]; 7(2):90-98. Disponível em: http://pepsic.bvsalud.org/pdf/rpsaude/v7n2/ v7n2a02.pdf.

35. Lussi IAO, Ferigato SH, Gozzi APNF, et al. Saúde mental em pauta: afirmação do cuidado em liberdade e resistência aos retrocessos. Cad. Bras. Ter. Ocup. 2019; 27:1-3. Disponível em: http://www.cadernosdeterapiaocupacional.ufscar.br/index.php/cadernos/ article/view/2502/0.

36. Almeida R. A onda quebrada - evangélicos e conservadorismo [internet]. Cad. Pagu. 2017 [acesso em 2020 jul 22]; 50. Disponível em: https://www.scielo.br/pdf/ cpa/n50/1809-4449-cpa-18094449201700500001. pdf.

37. Almeida R, Toniol R. Conservadorismos, fascismos e fundamentalismos: análises conjunturais. Campinas: Unicamp; 2018.

Recebido em 14/01/2020

Aprovado em 15/08/2020

Conflito de interesses: inexistente

Suporte financeiro: não houve 